\title{
Erratum to: Healthy living practices in families and child health in Taiwan
}

\author{
Yi-Ching Lin • Jennifer Chun-Li Wu $\cdot$ Shu-Ti Chiou • \\ Tung-liang Chiang
}

Published online: 26 February 2016

(c) Swiss School of Public Health (SSPH+) 2016

\section{Erratum to: Int J Public Health (2015) 60:691-698 \\ DOI 10.1007/s00038-015-0701-z}

In the original publication of the article, the Tables 1 and 2 values were incorrectly published. The correct Tables 1 and 2 are given below.

The online version of the original article can be found under doi:10.1007/s00038-015-0701-z.

Y.-C. Lin

College of Public Health, National Taiwan University, Room 621, No.17, Xu-Zhou Road, Taipei 10055, Taiwan

e-mail: vindalin@gmail.com

J. C.-L. Wu

College of Education, National Taipei University of Education, 134, Sec. 2, Heping E. Road, Taipei 10651, Taiwan

\section{S.-T. Chiou}

National Yang-Ming University, Health Promotion

Administration, Ministry of Health \& Welfare, Taipei, Taiwan

T. Chiang $(\varangle)$

College of Public Health, National Taiwan University, Room

620, No.17, Xu-Zhou Road, Taipei 10055, Taiwan

e-mail: tlchiang@ntu.edu.tw 
Table 1 Distribution of socioeconomic factors and Child Healthy Living Practices in Family (CHLPF) Index in Taiwan, 2008

\begin{tabular}{|c|c|c|c|c|c|c|}
\hline & \multirow{2}{*}{$\begin{array}{l}\text { Total } \\
(n=19,910)\end{array}$} & \multicolumn{5}{|c|}{ Child Healthy Living Practices in Family (CHLPF) Index } \\
\hline & & $\begin{array}{l}\text { Low } \\
(n=7070,35.5 \%) \\
n(\%)\end{array}$ & $\begin{array}{l}\text { Mid-low } \\
(n=6248,31.4 \%) \\
n(\%)\end{array}$ & $\begin{array}{l}\text { Mid-high } \\
(n=4444,22.3 \%) \\
n(\%)\end{array}$ & $\begin{array}{l}\text { High } \\
(n=1950,9.8 \%) \\
n(\%)\end{array}$ & $p$ value \\
\hline Sex & & & & & & 0.001 \\
\hline Boys & $10,363(52.6)$ & $3742(36.1)$ & $3340(32.2)$ & $2336(22.5)$ & $945(9.1)$ & \\
\hline Girls & $9349(47.4)$ & $3328(35.6)$ & $2908(31.1)$ & $2108(22.5)$ & $1005(10.7)$ & \\
\hline Family structure & & & & & & $<0.001$ \\
\hline Single-parent & $947(4.8)$ & $407(43.0)$ & $308(32.5)$ & $173(18.3)$ & $59(6.2)$ & \\
\hline Two-parents & $18,765(95.2)$ & $6663(35.5)$ & $5940(31.7)$ & $4271(22.8)$ & $1891(10.1)$ & \\
\hline Family income & & & & & & $<0.001$ \\
\hline Low & $2343(11.9)$ & $1206(51.5)$ & $648(27.7)$ & $350(14.9)$ & $139(5.9)$ & \\
\hline Middle & $10,172(51.6)$ & $3971(39.0)$ & $3181(31.3)$ & $2178(21.4)$ & $842(8.3)$ & \\
\hline High & $7197(36.5)$ & $1893(26.3)$ & 2419 (33.6) & $1916(26.6)$ & $969(13.5)$ & \\
\hline Maternal education & & & & & & $<0.001$ \\
\hline Junior high school and below & $2805(14.2)$ & $1417(50.5)$ & 811 (28.9) & $431(15.4)$ & $146(5.2)$ & \\
\hline Senior high school & $7881(40.0)$ & $3139(39.8)$ & $2452(31.1)$ & $1600(20.3)$ & $690(8.8)$ & \\
\hline Junior college & $4835(24.5)$ & $1469(30.4)$ & $1628(33.7)$ & $1214(25.1)$ & $524(10.8)$ & \\
\hline University and graduate & $4191(21.3)$ & $1045(24.9)$ & $1357(32.4)$ & $1199(28.6)$ & $590(14.1)$ & \\
\hline Paternal education & & & & & & $<0.001$ \\
\hline Junior high school and below & $2674(13.6)$ & $1354(50.6)$ & $792(29.6)$ & $396(14.8)$ & $132(4.9)$ & \\
\hline Senior high school & $7860(39.9)$ & $3219(41.0)$ & $2425(31.2)$ & $1551(19.7)$ & $636(8.1)$ & \\
\hline Junior college & $4164(21.1)$ & $1281(30.8)$ & $1372(32.9)$ & $1040(25.0)$ & $471(11.3)$ & \\
\hline University and graduate & $5014(25.4)$ & $1216(24.3)$ & $1630(32.5)$ & $1457(29.1)$ & $711(14.2)$ & \\
\hline Missing & 198 & & & & & \\
\hline
\end{tabular}


Table 2 Crude and adjusted odds ratio of Child Healthy Living Practices in Family (CHLPF) Index levels and mother rated health of children in Taiwan, 2008

Mother-rated health of children (rated "poor health")

Crude odds ratio

1.00 (ref)
0.79
0.62
0.47

1.00 (ref)

0.85

Girl

Family structure

Single-parent

Two-parent

Family income

Low

Middle

High

Maternal education

Junior high school and below

Senior high school

Junior college

University and graduate

Paternal education

Junior high school and below

Senior high school

1.00 (ref)

0.84

$0.72,0.97$

1.00 (ref)

0.82

0.68

$0.74,0.91$

$0.61,0.76$

1.00 (ref)

1.11

1.08

0.96

1.00 (ref)

0.93

0.86

0.83

$0.73,0.85$

$0.56,0.68$

$0.41,0.54$

$0.79,0.90$

Junior college

$0.99,1.23$

$0.85,1.08$
University and graduate

Adjusted odds ratio

$95 \% \mathrm{CI}$

1.00 (ref)

0.80

$0.73,0.86$

0.63

0.48

$0.57,0.69$

$0.42,0.56$

1.00 (ref)

0.85

$0.79,0.90$

1.00 (ref)

0.96

$0.82,1.12$

1.00 (ref)

0.82

$0.74,0.92$

0.69

$0.61,0.79$

1.00 (ref)

1.26

$1.13,1.41$

1.38

$1.12,1.58$

1.31

$1.13,1.53$

$95 \%$ CI $95 \%$ confidence interval 